\title{
Compresión del nervio mediano asociado a un proceso supracondíleo humeral y ligamento de Struthers. A propósito de un caso y revisión de la literatura
}

\author{
S. M. a Medina Macías, J. A. Medina Henríquez, A. Marcos García, \\ G. Muratore Moreno
}

Hospital UNIVERSITARIO INSULAR DE GRAN CANARIA

SERVICIO COT. UNIDAD DE MANo Y MIEMBRo SUPERIOR

Correspondencia:

Dra. Sonia Ma Medina Macías

C/. Alc. J. Hidalgo Carreño, 4

35100 S. Fernando-Maspalomas (Gran Canaria)

Tel.: 679192469

e-mail: soniamedina@comlp.es

Se han descrito hasta ochos estructuras anatómicas causas de compresión del nervio mediano en su recorrido, siendo muy raras las originadas por un proceso supracondíleo y ligamento de Struthers, que desde un punto de vista anatómico hay que diferenciarlo de la Arcada de Struthers, aunque no descrita por este autor, y sitio de compresión del nervio cubital en el codo.

En el síndrome de Struthers, a diferencia de la compresión del nervio mediano en otras localizaciones, el tratamiento quirúrgico inicial consigue la desaparición de la sintomatología neurológica en menos de un mes.

Palabras claves: Proceso supracondíleo, Ligamento de Struthers, Nervio Medinao.
Causes of compression of the median nerve in their route have been described until eight anatomical structures, being very rare the originated ones by the supracondilar process of the humerus and Struthers ligament, that from an anatomical point of view there is to differentiate it from the Arcade of Struthers, not described by this author, and site of compression of the ulnar nerve in the elbow.

In the syndrome of Struthers, unlike the compression of the median nerve in other locations, the initial surgical treatment obtains the disappearance of the neurological sintomatology in less of a month.

Key words: Supracondilar process of de humerus, Struthers ligament, Median nerve. 


\section{INTRODUCCIÓN}

Algunos carnívoros, en particular los gatos, presenta un canal supracondíleo característico constituido por un puente óseo, que teniendo su origen sobre la parte anteromedial de la extremidad distal del húmero, se extiende hasta el cóndilo medial, pasando por su interior de atrás hacia delante el nervio mediano y la arteria braquial ${ }^{1}$.

Aproximadamente 1/5 de los hombres en la actualidad poseen un vestigio de este canal, considerado como una manifestación en el humano de una característica primitiva ${ }^{1}$, descrito en 1848 por Struthers ${ }^{2}$ como un proceso del húmero que partiendo de su superficie anteromedial continuaba con una formación ligamentosa que acaba por insertarse en la epitróclea, y que se acompañaba de alteraciones neurológicas del nervio mediano, pues el proceso y el ligamento forman un pequeño orificio delimitado medialmente por el tabique intermuscular medial y por la superficie anterior de la epitróclea, a través del cual pasan la arteria braquial y el nervio mediano.

\section{CASO CLÍNICO}

Se presenta el caso de un varón de 14 años que acude por presentar dolor en la cara me-

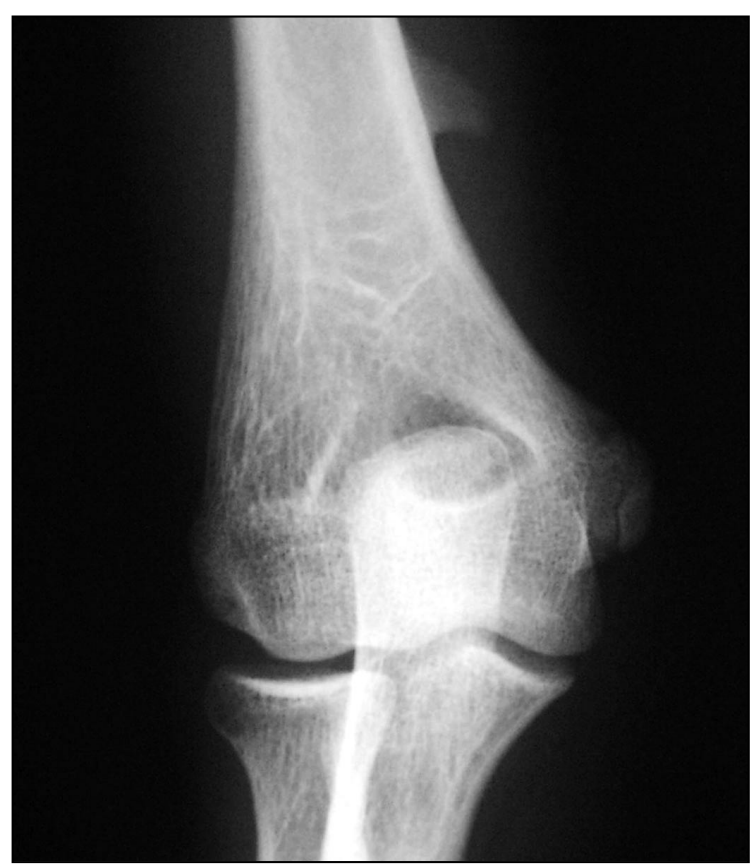

Figura 1: Rx AP del brazo derecho. Proceso supracondíleo.

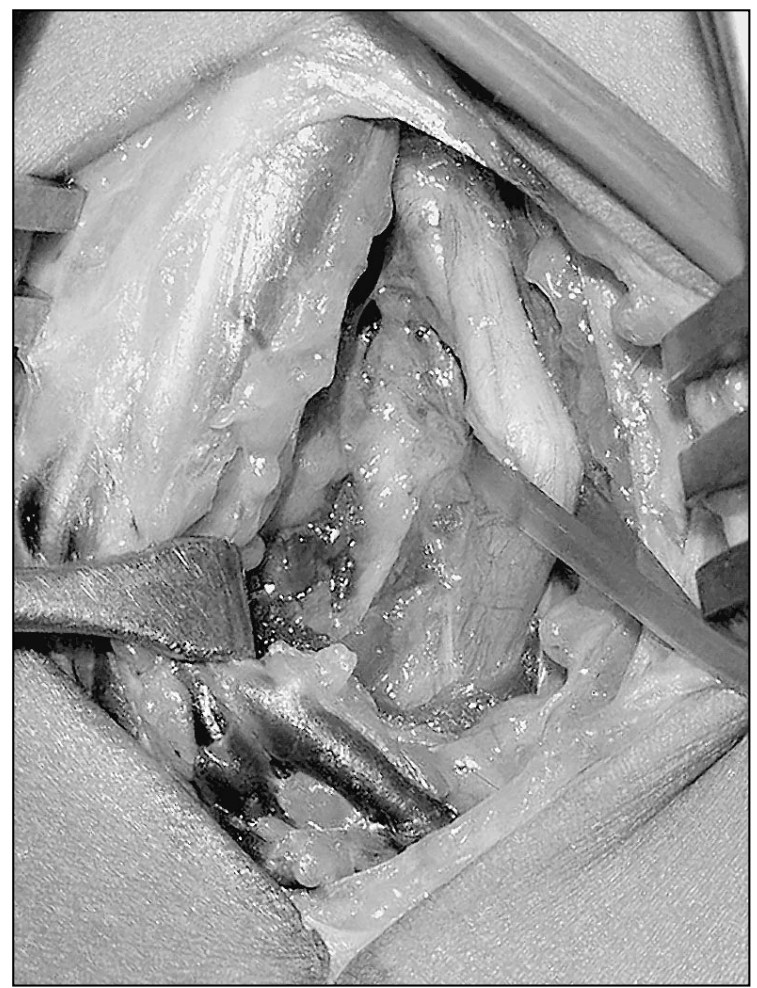

Figura 2: Nervio mediano y su relación con el proceso supracondíleo y el ligamento de Struthers (parte inferior).

dial del brazo derecho y ocasionalmente parestesias en el territorio del nervio mediano a nivel del antebrazo y la mano.

Se realiza una radiografía de ambos codos, encontrando en la cara interna del tercio distal del húmero derecho una excrecencia ósea (Figura 1), palpable y dolorosa a la palpación, observando además en la exploración clínica el aumento del dolor y de las parestesias con la flexión del codo, y a la compresión a ese nivel, sin que existiera déficit motor.

Ante la sospecha clínica y radiología de un Síndrome de Struthers, se interviene al paciente realizándose la liberación del nervio mediano, la sección del ligamento de Struthers, y la exéresis del proceso supracondíleo, tras lo cual se produjo la curación clínica del paciente (Figuras 2 y 3).

\section{DISCUSION}

Se han descrito hasta ochos estructuras anatómicas como causa de compresión del nervio mediano a lo largo de su recorrido, desde la axi- 


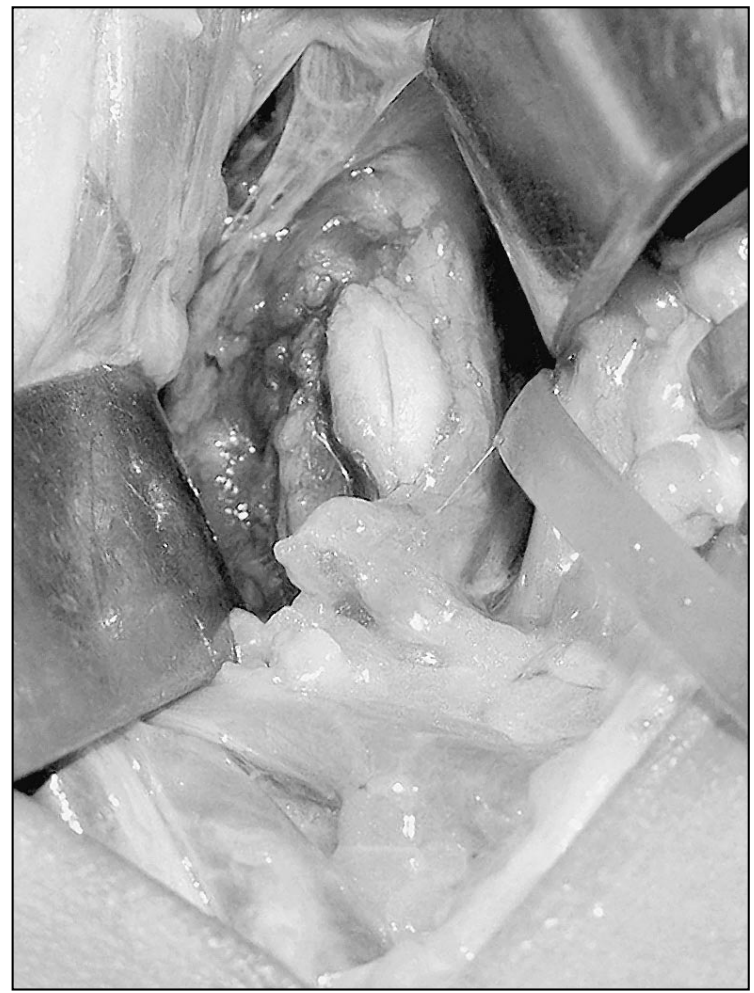

Figura 2: Nervio mediano y su relación con el proceso supracondíleo y el ligamento de Struthers (parte inferior).

la hasta la parte distal del antebrazo (músculo braquial, ligamento de Struthers asociado o no a un proceso supracondíleo, aponeurosis bicipital o lacertus fibrosus, pronador redondo, flexor superficial de los dedos, cabeza accesoria del flexor largo del pulgar (músculo de Gantzer) y estructuras vasculares) ${ }^{3}$, considerándose la presencia de un proceso supracondileo y un ligamento de Struthers una rara anomalía congénita que puede causar compresión del nervio mediano, de la arteria braquial o de ambos, cuadro clínico que se conoce como Síndrome de Struthers ${ }^{4}$, que es raro, encontrándose pocos casos descritos en la literatura ${ }^{5-10}$.

Desde un punto de vista anatómico, hay que diferenciar esta estructura de la denominada Arcada de Struthers, aunque se ha considerado incorrecto el uso de este término ${ }^{11}$, al comprobarse, tras las revisiones de varias publicaciones anatómicas, que no fue descrita por este autor; arcada que es un punto de compresión del nervio cubital en la región del codo y no del nervio mediano ${ }^{12}$.

En 1854 Struthers describió el ligamento braquial interno como un engrosamiento del tabi- que intermuscular interno ${ }^{13}$, siendo descrita posteriormente esta arcada por Kane en $1973^{14}$ y por Spinner en $1976^{15}$, como la región en la que el nervio cubital pasa del compartimento anterior al posterior, por lo que está situada en el compartimento posterior del brazo, a unos $8 \mathrm{~cm}$ (entre 4 y 10,5 cm) por encima del cóndilo medial, presentando como límite anterior el tabique intermuscular interno, y el origen tendinoso de una parte de las fibras superficiales de la cabeza medial del tríceps, y como suelo la parte interna del húmero y las fibras profundas de la cabeza medial del tríceps, arcada que es más o menos constante estando presente en un $75 \%$ (67 a 100\%) de la población. Contrariamente a la arcada, el ligamento de Struthers y el proceso supracondíleo se localizan en el compartimento anterior del brazo, más o menos a $5 \mathrm{~cm}$ por encima de la epitróclea, formando un orificio delimitado medialmente por el tabique intermuscular medial y la superficie anterior de la epitróclea, no estando presente en más del $1 \%$ de la población, siendo una de las causas de compresión del nervio mediano a nivel del codo $^{16}$.

El diagnóstico del Síndrome de Struthers es clínico y radiológico. Se presenta con dolor, alteraciones de la sensibilidad en la cara interna de brazo y antebrazo o mano y/o pérdida de la función motora del nervio mediano; en ocasiones también origina una compresión de la arteria braquial, lo que se pone de manifiesto ante la disminución de la intensidad del pulso radial $^{4,17-18}$. El proceso supracondíleo puede presentarse en diversos grados de desarrollo, pudiendo ser visible o no en la radiografía, donde aparece como una exostosis a nivel de la cara interna del húmero ${ }^{18}$.

Se puede encontrar un signo de Tinel + en el codo por encima de la compresión, pero la prueba más sensible es la presión directa a nivel del ligamento de Struthers, el estudio EMG contribuye poco al diagnóstico por su escasa sensibilidad $^{19}$.

La clínica, de aparición espontánea, aparece tras un esfuerzo, sobre todo en caso de sobreutilización del miembro superior (durante un esfuerzo intenso o en la práctica deportiva). Las manifestaciones clínica se pueden provocar tras una flexión del codo contrarresistencia más allá 
de los $120^{\circ}$, lo que nos puede ayudar a hacer el diagnóstico clínico diferencial con otras zonas de compresión del nervio mediano a lo largo de su recorrido (pronador redondo: flexión del codo con pronación del antebrazo contrarresistencia; lacertus fibrosus: supinación contrarresistencia; arco del flexor superficial: flexión contrarresistencia del $3^{\circ}$ dedo). El diagnóstico diferencial también hay que hacerlo dentro de un síndrome de compresión escalonada, con su posible asociación a un síndrome de canal carpiano o a un síndrome costoclavicular ${ }^{19}$.
A diferencia de la compresión del nervio mediano en otras localizaciones en los que se puede instaurar inicialmente un tratamiento conservador basado fundamentalmente en la supresión de la actividad nociva y la utilización de ortesis de reposo, en el caso del síndrome de Struthers con una exostosis ósea visible en la radiología, el tratamiento quirúrgico con resección de la misma y la sección del ligamento de Struthers, consigue la recuperación del paciente con desaparición de la sintomatología neurológica en menos de un mes ${ }^{18,20}$.
1. Oberlin C. Anatomie comparée de la main. Chir Main, 2006; 25: 111-8.

2. Struthers J. On a peculiarity of the humerus and humeral artery. Monthly J Med Sci, 1848; 8: 264-7.

3. Bilecenoglu B, Uz A, Karalezli N. Posible anatomic structures causing entrapment neuropathies of the median nerve: an anatomic study. Acta Orthop Belg, 2005; 71: 169-76.

4. Bilge $T$, Yalaman $O$, Bilge $S$, Cokneseli B, Barut S. Entrapment neuropathy of the median nerve at the level the ligament of Struthers. Neurosurgery, 1990; 27: 787-9.

5. Al-Naib I. Humeral supracondylar spur Struthers ligament. A rare cause of neurovascular entrapment in the upper limb. Int Orthop, 1994; 18: 393-4.

6. Mimoun M, Baux S, Kirsch JM, Kuhlmann JN, Nivesse D. Subepitrochlear apophysis. A sourse of median nerve compression. Review of the literature. Ann Chir Main, 1986; 5: 332-4.

7. Laha RK, Dujovny M, DeCastro SC. Entrapment of median nerve by supracondylar process of the humerus. Case re-

\section{BIBLIOGRAFÍA}

port. J Neurosurg, 1977; 46: 252-5.

8. Peyroux LM, Dunaud JL, Moughabghab M. Supraepithochlear process and median nerve compression. Ann Chir Main, 1991; 10: 68-70.

9. Smith RV, Fisher RG. Struthers ligament: a source of median nerve compression above the elbow. Case report. Neurosurgery, 1973; 38: 778-9.

10. Suranyi L. Median nerve compression by Struthers ligament. J. Neurol. Neurosurg. Psychiatry, 1983 ; 46: 1047-9.

11. De Jesus R, Dellon AL. Historic origin of the "Arcade of Struthers". J Hand Surg Am, 2003; 28: 528-31.

12. Siqueira MG, Martins RS. The controversial arcade of Struthers. Surg Neurol, 2005; 64 (S1): 17-21.

13. Struthers J. One some points in the abnormal anatomy of the arm. Br. Foreign Med Chir Rev, 1854; 12: 523-33.

14. Wehrli L, Oberlin C. The internal brachial ligament versus the arcade of Struthers: an antomical study. Plast Reconstr Surg, 2005; 115: 471-7.

15. Spinner M, Kaplan EB. The relationship of the ulnar nerve to the medial intermuscular septum in the arm ant its clinical significance. Hand, 1976; 8: 239-42.

16. Roulot E, Charlez C. Syndromes Canaliculaires du membre supérieur. Le nerf ulnaire au coude. Chir Main, 2004; 23: S110-7.

17. Aydinlioglu A, Cirak B, Akpinar F, Tosun N, Dogan A. Bilateral median nerve compression at the level of Struthers ligament. Case report. J Neurosurg, 2000; 92: 693-6.

18. Marchetti N, Bachechi P, Barbieri E, Guido G. Síndromes nerviosos Canaliculares de los miembros de etiología no traumática. Compresión del nervio mediano en el ligamento de Struthers. Padova: Liviana editrice, 1982: 31-33.

19. Allieu Y, Chammas M, Roux JL. Sindromes canalaires et des défiles (canal carpien excl.). Encycl. Méd. Chir (Elservier, Paris - France), Appareil locomoteur, 15005 A 10, 1997, 16 p.

20. Casadei R, Ferraro A, Ferruzzi A, Innao V, Mercuri M. Supracondylar process of the humerus: tour cases. Chir Organi Mov, 1990; 75: 265-77. 